\title{
AN ESE EVENT FOR PSR 0329+54
}

\author{
ANTHONY MINTER \\ National Radio Astronomy Observatory, P.O. Box 2, Green Bank, WV, 24944, U.S.A.
}

\begin{abstract}
Space Very Long Baseline Interferometry observations of PSR 0329+54 which, by luck, occurred during an interstellar fringing event, are presented. Separate images of the pulsar were not detected. However, the pulsar was observed to be extended. The size of PSR $0329+54$ during this event is $1.88 \times 1.67$ mas with a position angle of $30^{\circ}$ East of North. This could be due to two separate images of the pulsar separated by $\Delta \theta \lesssim 0.5$ mas or angular broadening. The observed image size is larger than the expected angular broadening size of $<0.1$ mas from the observations of Britton, Gwinn and Ojeda (1998).
\end{abstract}

\section{Introduction}

Extreme scattering events (ESEs) are thought to occur when a 'plasma lens' lies nearly along the line of sight to a radio source (Clegg, Fey and Lazio, 1998). It is generally believed that interstellar fringing in pulsar dynamic spectra are also the result an ESE event in which two or more dominant ray-bundles reach the Earth from the pulsar (Rickett, Lyne and Gupta, 1997). Under such conditions, two or more images of the pulsar should be present when mapped at an angular resolution of a few milli-arcseconds or less (Rickett, Lyne and Gupta, 1997). The observations presented in this paper are the first high angular resolution images made of a pulsar during an interstellar fringing event. Multiple images of PSR $0329+54$ are not observed. However, it is not a point source either.

\section{Observations}

The pulsar PSR $0329+54$ was observed on August 22, 1998 from $6^{h}$ to $18^{h}$ UTC using Space Very Long Baseline Interferometry (SVLBI) (Hiribayashi et al., 1998). The NRAO's 140 Foot telescope was also used to make simultaneous 'single dish' observations of PSR 0329+54 during the SVLBI observations. The single dish observations had a total bandwidth of $70 \mathrm{MHz}$ with a resolution of $0.078125 \mathrm{MHz}$. The SVLBI observations have a total bandwidth of $32 \mathrm{MHz}$ and a resolution of $0.125 \mathrm{MHz}$. The SVLBI observations were correlated using the 'pulsar gating' mode of the VLBA correlator. The gate had a width of $15 \%$ of the pulsar period and was centered on the center of the main pulse. 

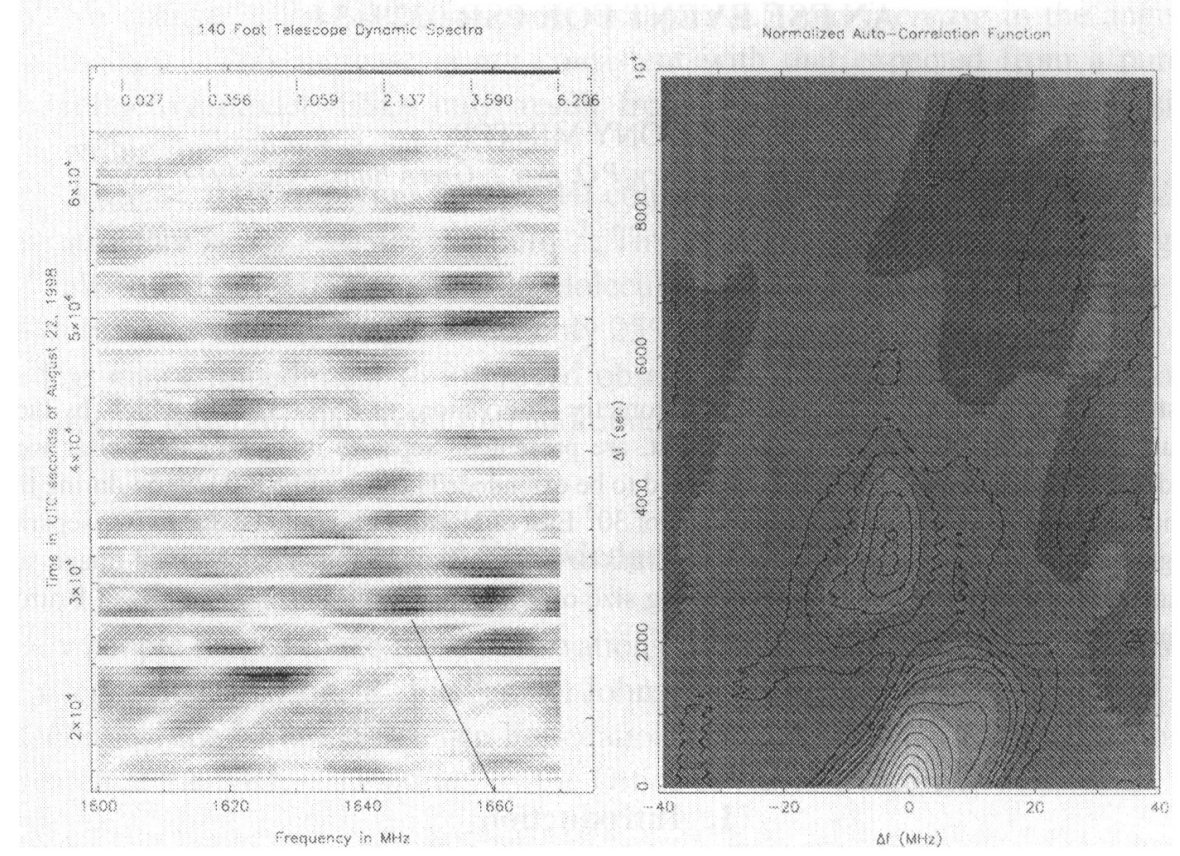

Figure 1. Dynamic spectra from the 140 Foot single dish data (a) and its normalized auto-correlation function (b). The line in the dynamic spectra indicates the direction of the fringes. The flux scale, in $\mathrm{Jy}$, is given in the upper portion of (a). Interference from the Iridium satellites is clearly visible in (a). The contours in (b) are $0.0,0.1,0.125,0.15,0.175,0.2,0.3,0.4,0.5,0.6,0.7,0.8$, and 0.9 .

\section{Interstellar Fringing Event}

The dynamic spectrum of the peak of the pulsar emission from the 140 Foot observations is shown in Figure 1a. The normalized auto-correlation function of the dynamic spectrum is shown in Figure $1 \mathrm{~b}$. The interstellar fringes can be seen as the periodic bright 'scintles' that go from the lower right to the upper left in Figure la. A solid line is shown in Figure 1a which indicates the direction of the fringes. The expected drift of the scintillation pattern is also obvious - being from the lower left to the upper right in Figure 1a.

The measured scintillation bandwidth and timescale during this observation were found to be $v_{d}=5.1 \pm 0.1 \mathrm{MHz}\left(\mathrm{HWHM}\right.$ at $\Delta t=0$ ) and $\tau_{d}=1100 \pm 50$ seconds ( $e^{-1}$ half width at $\Delta f=0$ ), respectively. The fringe periods for the peak of the pulse profile were determined to be $3223 \pm 170$ seconds along the time axis and $-3.18 \pm 0.06 \mathrm{MHz}$ along the frequency axis from the auto-correlation function. Since the fringe periods and the diffractive scintillation timescales and frequencies are similar in magnitude, it is not possible to use the 'secondary spectrum' (Rickett, Lyne and Gupta, 1997) to find the fringe peak since it is confused with the DISS. The auto-correlation function was thus used to determine the fringe periods since the fringe peak and the DISS are not confused in this function. Following the ar- 

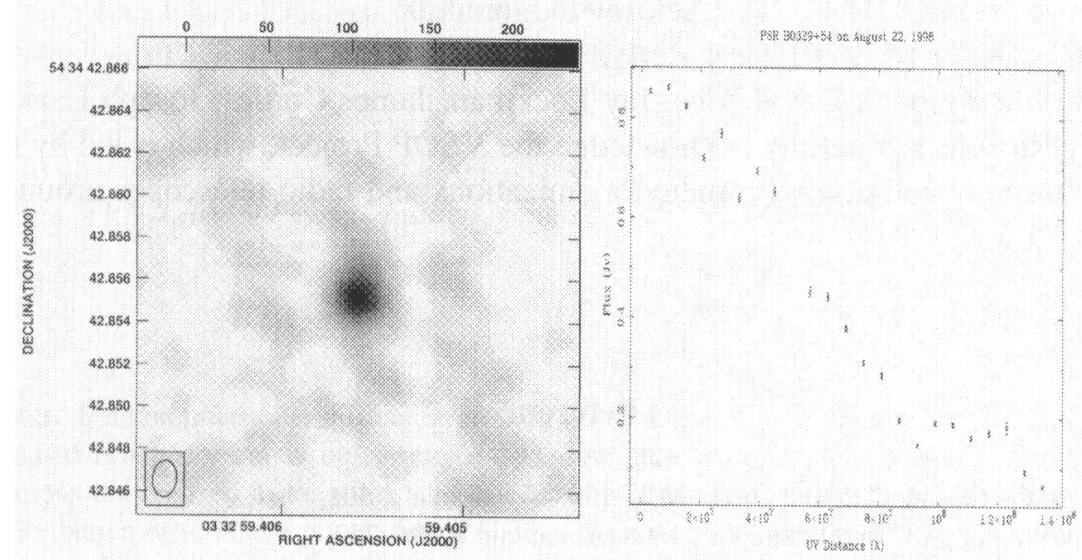

Figure 2. a) Image of PSR $0329+54$ from the Space-VLBI observations during the interstellar fringing event. The color bar indicates the flux in Janskies. The beam is shown in the lower left corner. b) Flux vs. UV distance determined from averaging within equally spaced radial bins in UV distance.

guments in Rickett, Lyne and Gupta and using $D \sim 1.43 \mathrm{kpc}$ (Taylor, Manchester and Lyne, 1993), it can be estimated that the multiple images producing the fringing during the August 22, 1998 PSR 0329+54 observations should have a separation $\Delta \theta \geq 0.35$ mas.

In Figure 2a, I show the image derived from the entire SVLBI data set after selfcalibration. The SVLBI data set has a resolution of $1.67 \times 1.12$ mas with a position angle of $-5.7^{\circ}$ (East of North). Figure $2 b$ shows the flux versus UV distance for the data set. Multiple images of PSR 0329+54 are not observed at this resolution. However, PSR $0329+54$ is not a point source either. A single elliptical Gaussian is able to provide a good fit to the image. The best fit elliptical Gaussian has a major axis of $1.88 \pm 0.01$ mas, a minor axis of $1.67 \pm 0.01$ mas and a position angle of $30^{\circ} \pm 3(\mathrm{E}$ of $\mathrm{N})$.

If the extended source size of PSR $0329+54$ is the result of multiple images of the pulsar which are not resolved then the image separation is $\Delta \theta \lesssim 0.5$ mas.

Britton, Gwinn and Ojeda (1998) determined that the angular broadening of PSR $0329+54$ was $<3.4$ mas at $330 \mathrm{MHz}$. Scaling this to $1650 \mathrm{MHz}$, the Britton, Gwinn and Ojeda angular broadening limits corresponds to $<0.1$ mas. If the extended source size of PSR $0329+54$ during the fringing event is due to DISS, then we are observing enhanced angular broadening during the ESE. This is what was observed for PKS 1741-038 during an ESE (Lazio, Fey and Gaume, 2000). 


\section{Acknowledgements}

I would like to thank David Nice, Jay Lockman, James Cordes, Joseph Lazio and Barney Rickett. I gratefully acknowledge the VSOP Project, which is led by ISAS in Japan in cooperation with many organizations and radio telescopes around the world.

\section{References}

Britton, M.C., Gwinn, C.R. and Ojeda, M.J.: 1998, Astrophys. J. 501, 101 L.

Clegg, A.W., Fey, A.L. and Lazio, T.J.: 1998, Astrophys. J. 496, 253.

Hirabayashi, H., Hirosawa, H., Kobayashi, H., et al.: 1998, Science 281, 1825.

Lazio, T.J.W., Fey, A.L. and Gaume, R.A.: this volume.

Rickett, B.J., Lyne, A.G. and Gupta, Y.: 1997, Mon. Not. R. Astron. Soc. 287, 739.

Taylor, J.H., Manchester, R.N. and Lyne, A.G.: 1993, Astrophys. J. Suppl. 88, 529. 